\title{
RESTORATION OF MOTION BLURRED IMAGES
}

\author{
Juwei Lu*, Eunice Poon \\ EPSON Edge, EPSON Canada Limited, \\ 3771 Victoria Park Avenue, \\ Toronto, Canada
}

\author{
K.N. Plataniotis \\ The Edward S. Rogers Sr. Department of \\ Electrical and Computer Engineering, \\ University of Toronto, Canada
}

\begin{abstract}
In this paper, we present several algorithms developed for restoration of motion blurred images. We begin with a singleimage based deblurring approach in the case of linear constant motion. This approach is a wavelet-based method with a novel $l_{p}$-norm regularization term. Due to the introduction of the wavelet and regularization techniques, the approach is rather robust against noise amplification during deconvolution. Then, we further developed a general multi-image based deblurring framework improved from recent works of [1]. The proposed framework is able to effectively take advantage of information contained in the multiple input images, even when they are blurred in the same direction - a case hard to be dealt with by traditional solutions. The proposed methods are evaluated on both simulated and real data, and the obtained experimental results indicate promising results.
\end{abstract}

\section{INTRODUCTION}

This paper includes two contributions. First, a single-image based deblurring algorithm is proposed. The deblurring algorithm can be considered an improved version of the so-called Landweber method [2]. This improvement is achieved by introducing two additional processes: denoising in the wavelet domain and regularizing in the spatial domain. The wavelet has been shown to be a very efficient tool for noise reduction, while the regularization not only suppresses noise amplification during deconvolution, but also reduces ringing artifacts. In particular, the regularization method is based on a novel $l_{p}$-norm form of total variation, which is a revised version of the regularization term recently introduced in [1]. As a result of the revision, the proposed regularization method is approximately equivalent to the standard total variation regularization when $p=1$, while equivalent to the so-called Tikhonov-Miller regularization when $p=2$. Taking a $p$ value within $(1,2)$ leads to a regularization strength in between, and this helps to avoid over-sharp or over-smooth results in some cases.

\footnotetext{
*The authors would like to thank Dr. Alex Rav-Acha for valuable comments and unselfish suggestions on the proposed methods.
}

Secondly, some researchers have shown that when multiple motion-blurred images are available, having different blur directions, image restoration can be improved substantially. Our further studies reveal that the performance improvement could be extended to the case of multiple images blurred in the same direction if an appropriate weighting mechanism is introduced to combine the restoration results of these images. This leads to a general multi-image deblurring framework. The extension has an important sense in practical applications, for example, a sequence of images taken by a slightly moving cell-phone camera are generally blurred in the same or similar direction.

\section{A WAVELET-BASED DEBLURRING METHOD WITH $L_{P}$-NORM REGULARIZATION}

Consider an image, $I$, blurred due to a linear constant camera motion. Assuming the imaging system is linear and shiftinvariant, the blurring process can be expressed as:

$$
I(x, y)=(O \otimes h)(x, y)+N(x, y)
$$

where $O$ is the original image, $h$ is the point spread function (PSF) characterized by the camera motion, $N$ is additive noise, and $\otimes$ denotes the convolution operator. Restoring $O$ from $I$ and $h$ involves a deconvolution process, which has led to a large amount of works [2,3]. The difficulties are due to the existence of the additive noise and a cutoff frequency of the PSF. The most straightforward solutions to the problem are direct methods in the frequency domain such as the inverse filter and the Wiener filter. However, better results are obtained usually with iterative methods in the spatial domain, such as the Van Cittert, Landweber, and Richardson-Lucy methods $[2,3]$. In this work, the deblurring algorithms are developed under the Landweber iterative framework. However, it is not difficult to see that they can be easily incorporated into other iterative methods.

\subsection{Regularizing in the spacial domain}

A typical iteration in the Landweber method is

$$
O^{n+1}=O^{n}+\beta \cdot h^{*} \otimes\left(I-O^{n} \otimes h\right)
$$


where $h^{*}(x, y)=h(-x,-y)$, and $O^{n}$ is the current estimate of the desired original image.

When the noise term $N$ is nonzero, regularization constraints are generally needed to avoid noise amplification and reduce ringing artifacts during deconvolution. Two widely used regularization terms are the so-called "Tikhonov-Miller" (TM) [4] and "Total Variation"(TV) [5]. In an iteratively deblurring framework, the TM regularization term is

$$
R_{T M}(O)=\Delta O=\frac{\partial^{2} O}{\partial x^{2}}+\frac{\partial^{2} O}{\partial y^{2}}
$$

which attempts to minimize the $l_{2}$-norm function,

$$
\int\|\nabla O\|_{2}^{2} d x d y, \text { where } \nabla=\left[\frac{\partial}{\partial x}, \frac{\partial}{\partial y}\right] .
$$

The limitation of such a regularization is that it may regularize too much, resulting smoothed edges. On the other hand, the TV minimizes the function, $\int|\nabla O(x, y)| d x d y$. This leads to a regularization term:

$$
R_{T V}(O)=\nabla \cdot\left(\frac{\nabla O}{|\nabla O|}\right),
$$

For the convenience in computation, we can consider a revised version of Eq.5,

$$
\hat{R}_{T V}(O)=\nabla \cdot\left(\frac{\nabla O}{\left|O_{x}\right|+\left|O_{y}\right|}\right),
$$

which optimizes the $l_{1}$-norm function,

$$
\int\|\nabla O\|_{1} d x d y=\int\left(\left|O_{x}\right|+\left|O_{y}\right|\right) d x d y
$$

a slightly different measure for the TV [6].

The advantage of the TV regularization is that it regularizes the images while preserving the edges. However, the method may lead to staircase effect and round corners. In this work, we attempt to combine the advantages of the TM and TV regularization methods, while avoid their respective shortcomings. To this end, a new regularization term is introduced here,

$$
R_{p}(O)=\nabla \cdot\left[\frac{p \cdot O_{x}}{\left|O_{x}\right|^{2-p}+\left|O_{y}\right|^{2-p}}, \frac{p \cdot O_{y}}{\left|O_{x}\right|^{2-p}+\left|O_{y}\right|^{2-p}}\right],
$$

which targets at minimizing the $l_{p}$-norm of a TV-like function,

$$
\int\|\nabla O\|_{p}^{p} d x d y=\int\left(\left|O_{x}\right|^{p}+\left|O_{y}\right|^{p}\right) d x d y
$$

$R_{p}(O)$ can be considered a revised version of the regularization term introduced recently by [1]. With the revision, it is not difficult to see that the proposed regularization method is equivalent to the revised total variation regularization (Eq.6) when $p=1$, while equivalent to the Tikhonov-Miller regularization (Eq.3) when $p=2$. Taking a $p \in(1,2)$ value leads to a regularization strength in between, and this helps to avoid over-sharp or over-smooth results in some cases.

Using Eq.8, we can obtain a new regularized Landweber method. In the method, the current estimate of the original image is updated by

$$
O^{n+1}=O^{n}+\beta \cdot\left(h^{*} \otimes\left(I-O^{n} \otimes h\right)-\eta \cdot R_{p}\left(O^{n}\right)\right),
$$

where $\eta$ is a parameter controlling the strength of regularization.

\subsection{Denoising in the wavelet domain}

Provided that the input images have low signal-noise ratios, the TV or TM regularization constraint may not be sufficient to suppress noise. In these cases, an efficient tool that can be used for further noise reduction is the wavelet. Compared to the Fourier domain, the wavelet domain has many good properties that are of importance to image deblurring and denoising [3]. For example, the wavelet coefficients are particularly efficient in representing spatially localized features such as singularities or edges, which extend over the entire Fourier domain.

In general, redundant wavelet transforms such as the $\grave{a}$ trous wavelet transform [7] are used for image restoration. Using such transforms, the image $O(x, y)$ can be decomposed as:

$$
O(x, y)=c_{J}(x, y)+\sum_{j=1}^{J} w_{j}(x, y)
$$

where $c_{J}(x, y)$ is the last smoothed band of $O(x, y)$ and $w_{j}(x, y)$ denotes the wavelet coefficients in the $j$-th scale. To suppress noise, we regularize the wavelet coefficients $w_{j}(x, y)$ by soft thresholding [8],

$$
\begin{array}{r}
\hat{w}_{j}(x, y)=\quad m_{j}+\operatorname{sign}\left(w_{j}(x, y)-m_{j}\right) . \\
\\
\kappa\left(\left|w_{j}(x, y)-m_{j}\right|-\frac{\sqrt{2} \sigma_{n}^{2}}{\sigma_{j}^{2}}\right),
\end{array}
$$

where $\sigma_{n}^{2}$ is the variance estimate of noise, $\left(m_{j}, \sigma_{j}^{2}\right)$ are the local mean and variance at the location $(x, y), \operatorname{sign}(\cdot)$ is the signum function, and $\kappa(z)$ is a thresholding function with $\kappa(z)=z$ if $z>0$, and $\kappa(z)=0$ otherwise. The goal of using $\left(m_{j}, \sigma_{j}^{2}\right)$ is to adapt the regularization to local spatial properties. Also, to reduce ringing artifacts, we also regularize the low frequency band, $c_{J}(x, y)$ following the recommendation of [9]. This can be done by employing a locally weighted Wiener filter,

$$
\hat{c}_{J}(x, y)=m_{J}+\frac{\sigma_{J}^{2}}{\sigma_{J}^{2}+\sigma_{n}^{2}}\left(c_{J}(x, y)-m_{J}\right) .
$$

After the regularization, the image $O(x, y)$ can be reconstructed by an inverse wavelet transform using $\hat{c}_{J}(x, y)$ and $\hat{w}_{j}(x, y)$. 
The computational complexity of the proposed method is the major concern when it is applied to practical tasks. To reduce the computational cost, denoising in the wavelet domain can be done only one time every $n \geq 1$ iterations. If we choose a relatively small $n$ value (compared to the iteration number), our experiments indicate that this may only degrade the restoration performance slightly.

\section{A GENERAL MULTI-IMAGE DEBLURRING FRAMEWORK}

Recently, some researchers such as [1] have shown that when multiple motion-blurred images are available, having different blur directions, image restoration can be improved substantially. Furthermore, we found that the performance improvement could be extended to the case of multiple images blurred in the same direction, if an appropriate weighting mechanism is introduced to combine the restoration results of these images. The premise behind is not difficult to see that the cutoff frequencies of the PSFs characterized by different extents of linear motions (even at the same direction) are different, and thus different frequencies are lost with different PSFs. Additional images blurred by even larger extents of motions are able to offer complementary information regarding the original image. Grouping them together essentially leads to a better restoration performance. However, to avoid that the impact of significant information is weakened by other relatively inferior information, different images should be paid with different attentions during the ensemble-based restoration process. Therefore, the key issue here is to introduce an appropriate weighting mechanism to combine the information provided by different images.

Consider a set of input images, $I_{m}(x, y), m=1, \cdots, M$, with each one being a blurred version of the same image $O(x, y)$ by a linear motion with magnitude $l_{m}$ and direction $\theta_{m}$. Take into account the $M$ images together, the update rule of the regularized Landweber method (Eq.10) is changed to

$$
\begin{aligned}
O^{n+1} & =O^{n}+\beta \cdot\left(\bar{L}-\eta \cdot R_{p}\left(O^{n}\right)\right), \text { where } \\
\bar{L} & =\sum_{m=1}^{M} \omega_{m} \cdot\left(h_{m}^{*} \otimes\left(I_{m}-O^{n} \otimes h_{m}\right)\right),
\end{aligned}
$$

$\omega_{m}=\frac{l_{m}^{-q}}{\sum_{m=1}^{M} l_{m}^{-q}}$ are the weights involved in the combination, $q$ is a parameter to adjust the values of the weights, and $h_{m}$ is the PSF characterized by the motion $\left(l_{m}, \theta_{m}\right)$. It is not difficult to see that the weights are inversely proportional to the motion magnitudes. Such a weighting mechanism is in agreement with the principle that the image with a smaller blur should play a more important role during the ensemblebased deblurring process.
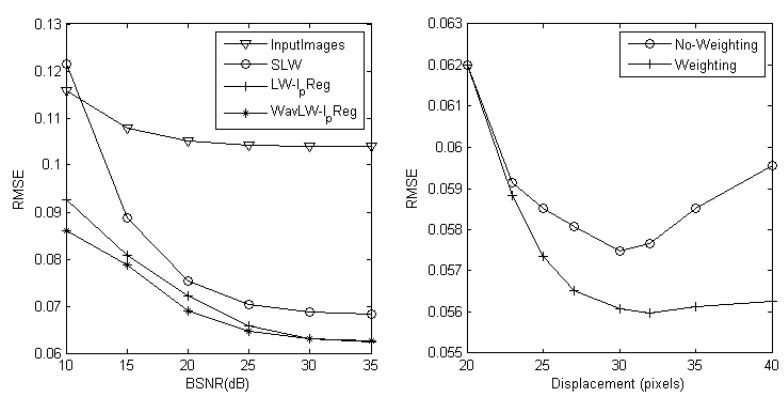

Fig. 1. Left: a comparison of single-image based methods. Right: a comparison of multi-image based methods. The 8 marked points on each curve correspond to the 8 input sets.

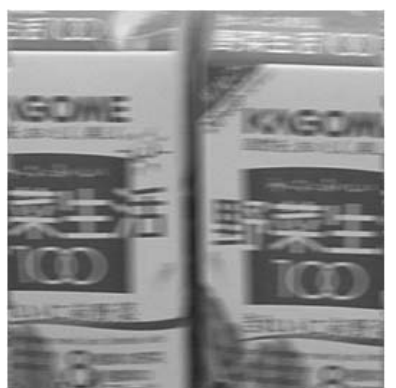

(A)

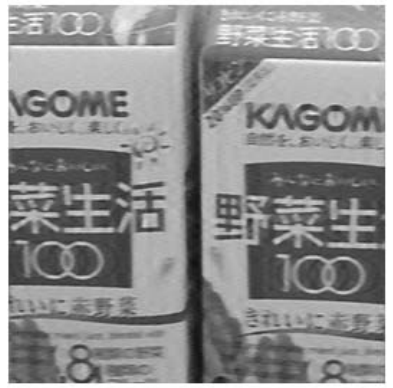

(C)

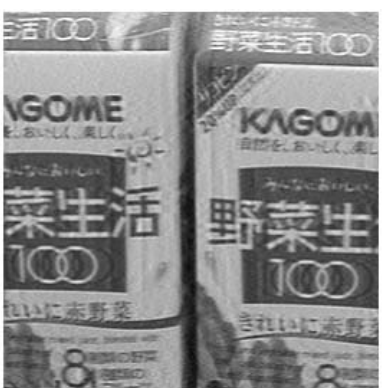

(B)

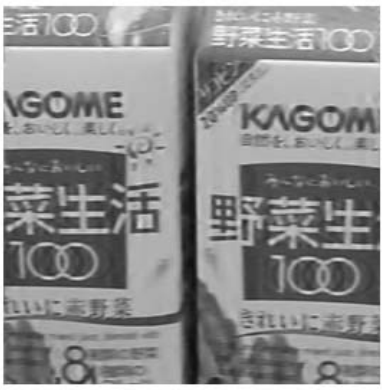

(D)
Fig. 2. An example of deblurring with single-image based methods. (A) The blurred and noisy input image with $l=20$, $\theta=0$ and BSNR=20dB. (B) Restored by SLW. (C) Restored by $\mathrm{LW}-l_{p}$ Reg. (D) Restored by WavLW-l $l_{p}$ Reg.

\section{EXPERIMENTAL RESULTS}

In the first experiment, we attempt to evaluate the effects of the two components introduced in section $2: l_{p}$-norm regularizing and wavelet denoising. To this end, three methods, the standard Landweber method (SLW), the Landweber method with $l_{p}$-norm regularization $\left(\mathrm{LW}-l_{p} \mathrm{Reg}\right)$, and the wavelet-based LW- $l_{p}$ Reg method (WavLW- $l_{p}$ Reg), are implemented and compared here. A blurred image is constructed by a simulated linear constant motion with $l=20$ and $\theta=0$ using the Paint Shop Pro 7 software. The Gaussian noise 
with variance $\sigma_{n}^{2}$ is then added onto the blurred image. A set of images are formed by changing the $\sigma_{n}^{2}$ values such that the blurred signal-to-noise ratios (BSNRs) of these images vary from $10 \mathrm{~dB}$ to $30 \mathrm{~dB}$. An example with $\mathrm{BSNR}=20 \mathrm{~dB}$ is shown in Fig.2:A. The three evaluated methods are applied to the set of blurred and noisy images, and the obtained rooted mean-squared error (RMSE) as a function of BSNRs are depicted in Fig.1:Left. For the two regularized methods, we set $p=1.25$ when $\mathrm{BSNR}<25 \mathrm{~dB}$ for a strong noise suppression, and $p=1$ otherwise. The noise variance $\sigma_{n}^{2}$ is estimated by using the robust median estimator in the highest subband of the wavelet transform of the input images [10]. It can be seen from Fig.1:Left that the SLW fails when the input data have a low BSNR. In contrast with this, both $\mathrm{LW}-l_{p}$ Reg and WavLW- $l_{p}$ Reg are rather robust against noise. Due to the introduction of an additional wavelet-based denoising process, the WavLW- $l_{p}$ Reg performs better than the LW- $l_{p}$ Reg, especially in the cases of low BSNRs. Furthermore, it can be observed that the wavelet-based process may not be necessary in the cases of BSNRs $>25 \mathrm{~dB}$, where the $l_{p}$-norm regularization alone seems to be sufficient to suppress the noise. An example that shows the deblurring performance of the three methods is also illustrated in Fig.2.

The second experiment is designed to demonstrate the effectiveness of the weighting mechanism introduced in the proposed general multi-image deblurring framework. Of particular interest is to observe the restoration performance when input images are blurred in the same motion direction. To this end, a set of 8 blurred images with $\theta=0$ and $l=$ $20,23,25,27,30,32,35,40$ are constructed in the same manner in the first experiment. No noise is added onto these images. Let $l_{i}$ denote the $i$-th image, $i=1, \cdots, 8$, sorted in an increasing order of motion blur extent. We then further form 8 sets, $L_{i}=\left\{l_{1}, \cdots, l_{i}\right\}, i=1, \cdots, 8$. Thus the set $L_{1}=\left\{l_{1}\right\}$ consists of only one image, while the set $L_{8}$ contains all the 8 images. Both the weighting and non-weighting solutions are applied to these sets. For the former, we set $q=2$ in the weight function $\omega_{m}(q, l)$. The obtained results are depicted in Fig.1:Right, where the horizontal axis corresponds to the motion extent of $l_{i}$ (i.e. the most blurred image) in the set $L_{i}$. Clearly, it can be seen from the results that both the weighting/non-weighting solutions are able to significantly improve the restoration performance when multiple blurred input images are available. However, more accurate restoration results may be biased by those less accurate ones if all the input images are treated with equal significance. It can be seen at this point that the weighting solution is much more efficient than the non-weighting one, especially when the input ensemble includes larger motion blurred images.

\section{CONCLUSIONS}

In this paper, we proposed a regularized wavelet-based image restoration method and a general multi-image deblurring framework. It can be seen that these proposed methods are general image restoration solutions, which only dependent on the current guess of the original image $O^{n}(x, y)$ or the motion parameters $\left(l_{m}, \theta_{m}\right)$. Therefore, in addition to the Landweber method, they can be employed to work with other iterative restoration methods, such as Richardson-Lucy. To further enhance the restoration performance, our future work will concentrate on developing an analytical method to estimate the optimal values of the parameters $p, q$.

In addition to the simulated data, the proposed methods are applied to motion blurred images in the real world. The PSFs are estimated from the motion parameters acquired by a gyro-based hardware system, which is designed by using the latest Epson angular velocity gyro (model: XV-3500CB) in the Epson Toronto research lab. The noise is not additive and Gaussian any more, and the motion is not limited to be linear constant. However, the obtained results indicate a high consistence with those observed in the experiments reported here.

\section{REFERENCES}

[1] Alex Rav-Acha and Shmuel Peleg, "Two motion-blurred images are better than one," Pattern Recognition Letter, vol. 26, pp. 311-317, 2005.

[2] J. BIEMOND, R. LAGENDIJK, and R. MERSEREAU, "Iterative methods for image deblurring," The Proceedings of the IEEE, vol. 78, no. 5, pp. 856-883, 1990.

[3] J. L. Starck, E. Pantin, and F. Murtagh, "Deconvolution in astronomy: A review," Publications of the Astronomical Society of the Pacific, vol. 114, pp. 1051-1069, October 2002.

[4] A. N. Tikhonov and V. Y. Arsenin, Solution of ill-posed problems, Winston-Wiley, New York, 1977.

[5] L. Rudin, S. Osher, and E. Fatemi, "Nonlinear total variation based noise removal algorithms," Physica D, vol. 60, pp. 259268, 1992.

[6] Y. Li and F. Santosa, "A computational algorithm for minimizing total variation in image restoration," IEEE Transactions on Image Processing, vol. 5, no. 6, pp. 987-995, 1996.

[7] J. L. Starck, F. Murtagh, and A. Bijaoui, Image Processing and Data Analysis: The Multiscale Approach, Cambridge University Press, Cambridge, 1998.

[8] D. L. Donoho, "De-Noising by soft thresholding," IEEE Transactions on Information Theory, vol. 41, no. 3, pp. 613-627, 1995.

[9] G. S. Shin and M. G. Kang, "Ringing artifact reduction in the wavelet-based denoising," in Proceedings of the IEEE International Conference on Acoustics, Speech, and Signal Processing, May 2004, vol. 3, pp. 233-236.

[10] S. G. Chang, B. Yu, and M. Vetterli, "Image denoising via lossy compression and wavelet thresholding," IEEE Transactions on Image Processing, vol. 9, pp. 15321546, 2000. 